\title{
Emergency department chest and abdominal computed tomography: impact on patients' management
}

BY MATTEO BRAMBATI, FIORANGELA MARLETTA, ROBERTO LA ROCCA, STEFANO PAGLIA

\section{Abstract}

Objective. The introduction of computed tomography (CT) has had a very significant impact on patient evaluation and management decisions in the Emergency Department (ED). One of the few published studies demonstrated a positive CT yield in $67 \%$ of patients admitted to hospital. The same study demonstrated that in $25 \%$ of cases, the CT examination was able to identify a pathological process unidentified by the ED team. Only one study has focused on the evaluation of the variation in patient management that each CT examination is able to achieve. The purpose of our study is to document the impact that CT has on patient diagnosis and management in the ED, employing a large patient series.

Materials and methods. The diagnostic and therapeutic impact of CT was evaluated in a consecutive series of 300 patients who were referred to the radiology department for a chest or abdominal CT examination as requested by the emergency physician. 
Results. The net impact of performing a CT was a change in planned treatment in 202 cases out of $300(67 \%)$ and proceding with the planned treatment in the remaining 98 cases (33\%). The only intention to demand a CT examination was significantly bound $(\mathrm{p}=0.015$, confidence interval 0.16 and 0.38 ) to a change in the planned treatment before CT (as it is established with Mc Nemar test).

Discussion. Our study showed that more than $67 \%$ of CT reports were able to change the course of treatment planned before CT execution and that in $56 \%$ of these cases, the execution of a CT examination was able to generate a major variation in treatment plan.

Key words: emergency department, computed tomography, chest, abdomen, clinical impact

\section{Introduction}

The introduction of computed tomography (CT) has had a very significant impact on patient evaluation and management decisions in the Emergency Department (ED). In the last twenty years, the number of CT examinations performed in the ED has increased with the proliferation of CT machines. $(1,2)$ One of the few published studies has demonstrated that a positive CT yield was found in $67 \%$ of patients admitted to hospital. (3) The same study has demonstrated that in $25 \%$ of cases, the CT examination was able to identify a pathological process unidentified by the ED team. (3) Another study has showed that a pre CT diagnosis was concordant with the discharge diagnosis in $37 \%$ of cases (4) and that a CT examination had the greatest impact on modifying the therapeutic approach in patients with suspected appendicitis, obviating the need for admission in $28 \%$ of cases. (4)

In 2010 the American College of Radiology published a review entitled 'Appropriateness Criteria Overview'. (5) This review intended to provide general guidelines on the basis of which every doctor should choose the type, the sequence and mode of execution of each radiological examination. A part of the review specifically addressed all the typical 
scenarios found in an ED.

According to data from a second study that analysed the typologies of patients who had undergone at least one CT scan in EDs in the USA and Canada (6), it is possible to say that the scenarios analyzed by the American College of Radiology cover most of the possible clinical questions asked by an ED physician.

Many studies concerning the increase in the number of diagnostic tests conducted in the ED have been performed, but only a few have focused on the evaluation of patient's management variation that each CT examination is able to achieve.

The purpose of our study is to document the impact that CT has on patient diagnosis and management in the ED, employing a large patient series. The large number of patients has allowed us to analyze the impact of $\mathrm{CT}$ with high degree of precision. We now report our experience from 300 consecutive CT referrals performed in our ED for patients presenting with acute, non-traumatic chest and abdominal pain.

\section{Materials and methods}

\section{Study design and population}

Institutional review board approval was received for this retrospective analysis. This study started in October 2012 and ended in March 2013. The diagnostic and therapeutic impact of CT examination was evaluated in a consecutive series of 300 patients who were referred to the radiology department for a chest or abdominal CT as requested by the emergency physician in our institution, an urban academic medical centre that sees approximately 40,000 patients per year. The interns working in the ED were from the departments of internal medicine and surgery. All CT examinations were ordered using a computer order entry system. Prior to completing the order for the CT, each physician was required to answer two questions. The questions were presented as part of the order entry 
process. The ordering physician was required to answer these questions before the CT was performed. Data were collected 24 hours a day, 7 days a week. No physicians refused to answer these questions and $100 \%$ compliance was noted.

\section{Questions}

Question I: Please select from the following list your most likely diagnosis: aortic aneurism, appendicitis, urinary tract stone, cholecystitis, diverticulitis, pulmonary embolism, malignancy, bowel obstruction/perforation, pancreatitis, pneumonia, pleural effusion or other. If "other" was selected, the physician was given the option of using free text entry to indicate his/her most likely diagnosis.

Question II: If you had to treat this patient without the benefit of CT, what would you consider the most appropriate management?

1. Admit the patient to a surgical department.

2. Admit the patient to a medical department.

3. Observe the patient in the ED.

4. Discharge the patient.

\section{Post CT analysis}

Each CT was interpreted by an attending radiologist within 30 minutes. Following the CT, each patient's medical record was reviewed and the result of the CT and the patient's post-CT management were archived in a tabular form. In case a difference between the treatment assumed prior to the $\mathrm{CT}$ and post $\mathrm{CT}$ examination was found, the clinician that ordered the CT examination was asked to classify the impact of the CT report using a predefined questionnaire. The questionnaire let the emergency physician choose one of the following five definitions for each CT report: not reliable (level o), not confirming clinical diagnosis (level 1), confirming clinical diagnosis (level 2), confirming and adding new information to clinical diagnosis (level 3), change of diagnosis (level 4). Furthermore, it was defined as a major change each CT report classified 
as level 1 or 4 and as a minor change each CT report classified as level o, 2 and 3 .

\section{Results}

The average age of our patients was $63 \pm 17$ years (range 14-92 years): 177 (59\%) were men, 123 (41\%) were women. Seventy-three chest CTs (24\%), 178 abdominal CTs (60\%) and 49 chest and abdominal CTs (16\%) were performed. The four most common pre-CT diagnoses were: bowel obstruction/perforation 55 of 300 (18\%), urinary tract stone 52 of 300 (17\%), pulmonary embolism 47 of 300 (16\%) and aortic aneurysm 34 of 300 (11\%). In addition to the 11 diagnostic choices, clinicians chose "other" and used the free text option to enter a diagnosis not offered in the alphabetical list in 17 of 300 (6\%) of cases. The complete disease spectrum is shown in table 1 . Prior to CT, the management plan included hospital admission for 150 patients (50\%), observational period in ED for 85 patients (28\%) and discharge for 65 patients (22\%). Prior to CT, 78 patients (52\%) were supposed to be admitted to a medical department and 72 patients (48\%) were supposed to be admitted to a surgical department. Following CT, 211 patients were actually admitted (70\%), 3 patients were detained in the ED for a prolonged observation period (1\%) and 86 patients were discharged (29\%). Following CT, 96 patients (45\%) were admitted to a medical department and 115 patients (55\%) were admitted to a surgical department (table 2). Thus, the net impact of performing a CT was a change in planned treatment in 202 cases out of $300(67 \%)$ and adhering to the planned treatment in 98 cases out of 300 (33\%). The only intention to demand a CT examination was significantly bound ( $\mathrm{p}=0.015$, confidence interval 0.16 and 0.38 ) to a change in the planned treatment before CT (as it is established with Mc Nemar test). The impact distribution of CT reports, that were able to change the therapeutic plan (202), was as follows: no report was not reliable (o\%), 48 reports (24\%) were not able to confirm the clinical diagnosis, 35 reports (17\%) were able to confirm the clinical diagnosis, 55 reports (27\%) were able to confirm the clinical diagnosis, adding new information, and 64 reports (32\%) were able to change the assumed 
diagnosis (figure 1). Hence, a major change in planned treatment was scored in 112 cases (56\%) and a minor change in planned treatment in 90 cases (44\%) (table 3).

\section{Discussion}

Patients presenting to the ED with complaints of acute non-traumatic abdominal or chest pain can pose a diagnostic challenge, as many diseases have overlapping presentations. During the past decade the practice of using plain abdominal and chest radiographs has been replaced with ultrasound and CT. Recent technical developments (multidetector row CT) have led to an increase in the use of CT in the ED, (7) have greatly enhanced CT's ability to accurately discriminate between those patients with a normal or abnormal abdomen or chest and have further characterized the etiology of the patient's abdominal or chest pain. (8) These technical developments have led to an increased use of CT in the ED, (1) for both non-traumatic and traumatic abdominal conditions. (9) And an increase in the amount of health care dollars spent on imaging. Thus, the utilization of computed tomography for acute, non traumatic chest or abdominal pain is a focus of much concern among health care administrators. Increasing funds are being spent on imaging patients with acute chest or abdominal pain despite the fact that the cost of the individual CT examinations is decreasing. (10) However, this debate has often failed to account for the clinical practice parameters that drive this increase in utilization. Our findings demonstrate that the mere execution of CT examinations is an important factor in determining admission to hospital. Our study shows that even after clinical evaluation, CT still adds additional information required for the correct diagnosis and triage of patients.

We found strong statistical correlation between conducting a CT examination and a change in treatment planned before the CT. This finding should not be considered surprising as a greater variation in therapeutic procedures is connected to emergency CT examination, especially if compared to CT scans performed routinely. In addition, a CT 
emergency examination performed only to confirm (or disprove) a diagnostic hypothesis already supported by strong clinical data, and therefore unable to change what has been already planned for the patient, should be considered a waste of economic resources.

Based on analysis of all 300 cases, we have shown that two-thirds of radiological investigations (202 cases or 67\%) favoured a therapeutic shift. This finding appears to be in contrast with the analyzed literature that shows a maximum rate of $28 \%$. (4) This result refers to a study in which the authors evaluated the percentage of variation of the planned treatment for each type of diagnostic question analyzed. The cited study included a larger number of patients (495), with a mean age of 51 years (standard deviation 20 years), examined only for non traumatic abdominal disorders and $28 \%$ represents the rate of patients, investigated for suspected appendicitis, who avoided hospitalization due to CT examination. The same article shows the raw data from which the conclusions were drawn. Analyzing these data, it has been estimated that 160 patients did not change the suggested therapeutic procedure (32\%), while 335 patients changed the suggested therapeutic procedure (68\%). Despite the substantial difference in the number of analysed cases, the average age and the explored region, the data can be considered overlapping. What we have demonstrated can be considered an objective reading of the daily reality that should satisfy ED doctors, especially if compared with the results obtained from other emergency departments.

Also, by narrowing the area only to CT examinations that are able to alter the planned therapeutic procedure, the results differ in a minimal way and clearly support what has been established. In fact, in $56 \%$ of cases, a major variation in planned treatment occurred, whereas a minor change occurred in the remaining cases.

\section{Limitations}

In this study, the role of ED CT in the management of patients with acute non-traumatic abdominal or chest pain was evaluated. For this purpose, 
a randomized, controlled trial powered to show the effect of CT in terms of patient outcome would be the best choice. However, these studies are long and costly and are somewhat unethical, implying an experimental arm of patients undergoing CT and a control arm of patients not undergoing CT even in the presence of a clinical indication. As a surrogate approach, a retrospective study was designed based on a survey of clinicians' opinions regarding CT reports. Using a predefined questionnaire, the impact of the CT was evaluated in terms of change of diagnosis and or therapy suggested.

\section{Conclusions}

In conclusion, this retrospective study demonstrates the efficacy of performing abdominal $\mathrm{CT}$ in the ED for patients with non traumatic chest and abdominal pain. Our study also showed that more than $67 \%$ of CT reports were able to change the treatment planned before CT execution and that in $56 \%$ of cases the execution of CT examination was able to generate a major variation in treatment plan. These results confirm the importance of CT examination in the ED setting and demonstrate its impact on therapy planning and change of diagnosis. In turn, CT execution is a predictor for hospital admission or discharge from the ED.

\section{References}

1. Sosna J, Slasky S, Bar-Ziv J. Computed tomography in the emergency department. Am J Emerg Med 1997 May;15(3): 244-7.

2. Broder J, Warshauer DM. Increasing utilization of computed tomography in the adult emergency department, 2000-2005. Emerg Radiol 2006 Oct;13(1): 25-30.

3. Modahl L, Digumarthy SR, Rhea JT, Conn AK, Saini S, Lee SI. Emergency department abdominal computed tomography for nontraumatic abdominal pain: optimizing utilization. J Am Coll Radiol 2006 Nov;3(11):860-6. 
4. Rosen MP, Siewert B, Sands DZ, Bromberg R, Edlow J, Raptopoulos V. Value of abdominal CT in the emergency department for patients with abdominal pain. Eur Radiol 2003 Feb;13(2):418-24.

5. ACR Appropriateness Criteria overview. Reston, VA: American College of Radiology; 2010.

6. Berdahl CT, Vermeulen MJ, Larson DB, Schull MJ. Emergency Department Computed Tomography Utilization in the United States and Canada. Ann Emerg Med 2013 Nov;62(5):486-94.

7. Powers RD, Guertler AT. Abdominal pain in the ED: stability and change over 20 years. Am J Emerg Med 1995 May;13(3):301-3.

8. Siewert B, Raptopoulos V, Mueller MF, Rosen MP, Steer M. Impact of CT on diagnosis and management of acute abdomen in patients initially treated without surgery. AJR Am J Roentgenol 1997 Jan;168(1):173-8.

9. Weishaupt D, Grozaj AM, Willmann JK, Roos JE, Hilfiker PR, Marincek B. Traumatic injuries: imaging of abdominal and pelvic injuries. Eur Radiol 2002 Jun;12(6):1295-311. Epub 2002 Apr 20.

10. Saini S, Sharma R, Levine LA, Barmson RT, Jordan PF, Thrall JH.

Technical cost of CT examinations. Radiology 2001 Jan;218(1):172-5.

Figure 1. Evaluation of clinical impact.

\section{Evaluation of clinical impact}

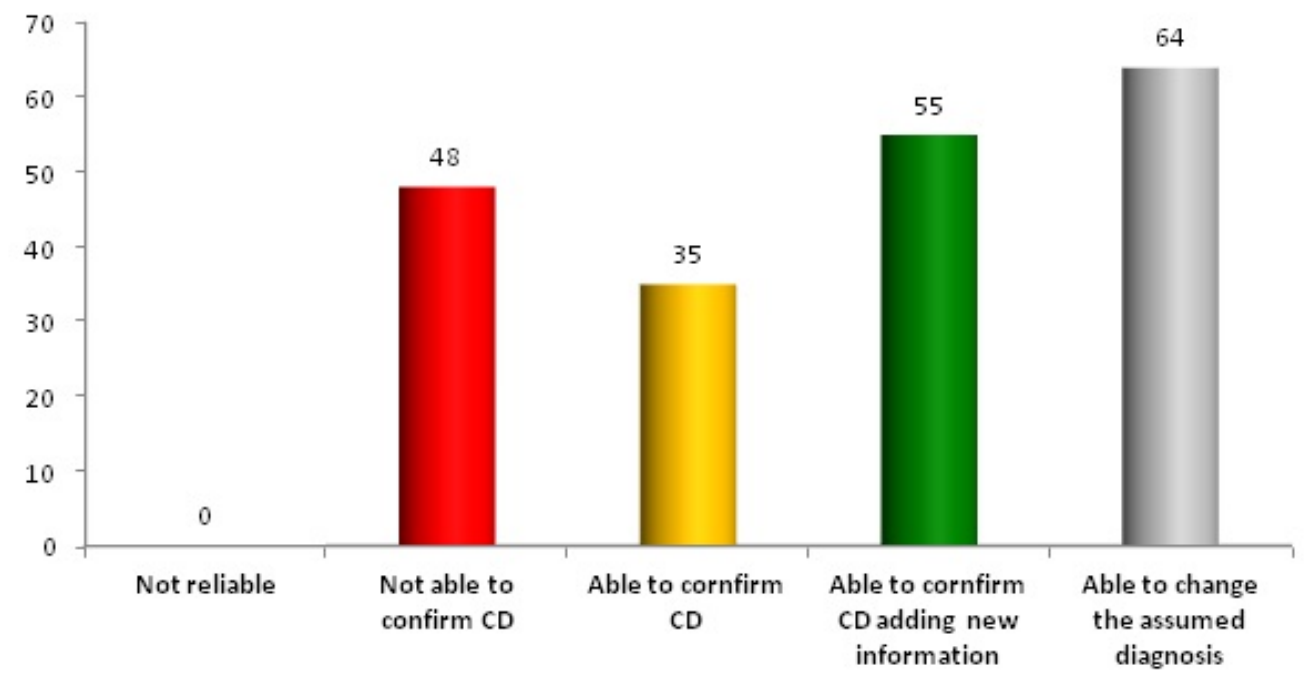

$\mathrm{CD}$, clinical diagnosis; CT, computed tomography.

Table 1. Complete disease spectrum. 
SIGNA VITAE 2016; 11(1):

\begin{tabular}{lll} 
Pre CT diagnosis & \multicolumn{2}{l}{ Frequency Percentage } \\
\hline Aortic aneurysm & 34 & $11 \%$ \\
\hline Appendicitis & 9 & $3 \%$ \\
\hline Urinary tract stone & 52 & $17 \%$ \\
\hline Cholecystitis & 26 & $8 \%$ \\
\hline Diverticulitis & 12 & $4 \%$ \\
\hline Pulmonary embolism & 47 & $16 \%$ \\
\hline Malignancy & 20 & $7 \%$ \\
\hline $\begin{array}{l}\text { Bowel } \\
\text { obstruction/perforation }\end{array}$ & 55 & $18 \%$ \\
\hline Pancreatitis & 5 & $2 \%$ \\
\hline Pneumonia & 15 & $5 \%$ \\
\hline Pleural effusion & 8 & $3 \%$ \\
\hline Other & 17 & $6 \%$ \\
\hline
\end{tabular}

CT, computed tomography.

Table 2. Comparison of planned treatment before computed tomography (CT) and actual treatment after CT examination.

\begin{tabular}{lllc}
$\begin{array}{l}\text { Planned } \\
\text { treatment }\end{array}$ & \multicolumn{3}{c}{ Discharged Observation Admitted } \\
\hline Pre CT & 65 & 85 & 150 \\
\hline Post CT & 86 & 3 & 211 \\
\hline Total & 151 & 88 & 361
\end{tabular}

Table 3. Evaluation of change in planned treatment.

Evaluation of change in planned treatment

Major change
Frequency Percentage

112 $55 \%$ 
Matteo Brambati, Stefano Paglia

Unità Sanitaria Complessa di Pronto Soccorso, Azienda Ospedaliera della provincia di Lodi, Lodi, Italy

Fiorangela Marletta, Roberto La Rocca

Unità sanitaria semplice di Pronto Soccorso/Dipartimento Emergenza e Accettazione, IRCCS Policlinico San Donato, San Donato Milanese, Italy

Corresponding author:

Matteo Brambati

Unità Sanitaria Complessa di Pronto Soccorso

Azienda Ospedaliera della provincia di Lodi

Via Kennedy 18, 20097

San Donato Milanese (MI), Italy

Phone number: +39 328-80 28910

E-mail address: brambati@hotmail.it

\section{Article printed from Signa Vitae: http://www.signavitae.com \\ URL to article: http://www.signavitae.com/2016/o5/emergency- department-chest-and-abdominal-computed-tomography- impact-on-patients-management/}

Copyright (C) 2015 Signa Vitae. All rights reserved. 